\title{
Weisthum und Gerichtsordnung der Gemeinde Ellerstadt vom Jahr 1555.
}

Seitdem Jacob Grimm zuerst auf die Bedeutung der Weisthümer hingewiesen hat, ist für die Sammlung und Veröffentlichung derselben so viel geschehen, dass die Publicirung neuer nicht mehr in allen Fällen für statthaft gelten kann. Denn die meisten dieser Rechtsquellen, wenigstens die aus einer und derselben Gegend, ähneln sich so sehr, dass der Gewinn, der aus der Mittheilung weiterer ungedruckter, deren ohne Zweifel eine ziemliche Anzahl noch zu finden wäre, gewöhnlich erwächst, ein sehr fragwürdiger ist. Der Abdruck der gegenwärtigen, so viel ich sehen kann, bisher noch nicht veröffentlichten Urkunde wird sich aber wohl damit rechtfertigen lassen, dass die Gerichtsordnung namentlich die charakteristischen Merkmale des Uebergangs der Receptionsepoche in so deutlicher Ausprägung zeigt wie wenig andere derartige Verfassungen dieser Zeit. Wie die Handschrift selbst besagt, ist diese Gerichtsordnung zwar eine Erneuerung der alten, aber sie ist zugleich zum guten Theil durch Zusätze, die aus dem neuen Recht herübergenommen wurden, erheblich vermehrt und wesentlich verändert, wie auch das Weisthum, dessen älteren Kern Grimm in seine Sammlung mit aufgenommen hat (I pag. 788), jetzt viele Zusätze erhalten hat. -

Das Original habe ich im Gemeindearchiv von Ellerstadt, einem Dorfe etwa 2 Stunden östlich von Dürkheim in der Rheinpfalz, aufgefunden. Es besteht aus 22 Pergamentblättern Fol., die durch die Buchstaben A bis X bezeichnet werden, und einem Umschlage, auf dem die Aufschrift und die ausgemalten Wappen der Affenstein und Flersheim angebracht sind. Dem regen Sinn der ländlichen Bevölkerung am Bunten entsprechend hat der Schreiber durchweg an rother und blauer Farbe nicht gespart. Von der in der Mitte durchgezogenen Seidenschnur ist noch ein Rest vorhanden; die daran befestigt gewesenen Siegel aber fehlen jetzt. -

Erneuwerung des $\beta$ wejsthumbs und der \|| gegebenen gerichtsordenung desß dorfs und $\|$ der gemeinden zuh Ellerstatt durch die gestrengen 
und edlen herren Wolffen von $\|$ Affenstein ${ }^{1}$ ) ritter, derselben zeit curfurstlicher Pfalntz hoffrichter ${ }^{2}$ ) etc. und dann Friderichen von Flerßhaeim ${ }^{3}$ ) den iungeren, beide gemelts dorfs rechte gerichts und oberherren, aufgericht, verordenet unde ubergeben im ior nach der geburt Jhesu Christi unßers erloeßers und seligmachers 1555.

A. Wir Wolff von Affenstein $\|$ ritter, der churfurstlichen Pfalntz hoffrichter etc. \| und Friderich von Flerßheim der iunger etc. \| bekhennen und thund khunt hiermit offent $\|$ lich gegen aller menigklichen, das wier, als des dorfs Ellerstatt ober und gerichts herren einmundigklich uns verglichen haben nochvolgent weisthumb und gerichtsordenung unsern underthanen und angehörigen zu Ellerstatt zu geben, zu ordnen und zuzustellen, wie wier auch ihnen vonn obrigkhaeit wegen hiermit zustellen und verordenen, der ursach, dieweil bißhero mancherlei alte ondugkliche misßbreuch und ihebungen bei inen gewesen welche dann wieder gemeine rechtliche ordenungen und process, dardurch nit allein die partheien in onnutzen verderblichen costen gefurt, sonder auch der richter etwan wiedersinns zu urtheilen bewegt worden und also underweilen den partheien ir gepurlich recht verhalten und gemeinlich alle handlungen gantz nichtigklich gehandlet befunden worden. Solches, sovil an uns zuvorkhomen und das hinfurter gemeltem unserm dorf zu Ellerstatt ein ordenlicher process durch die gerichtspersonnen und partheien (: so sich in rechtfertigung doselbst begeben:) gebraucht werde, so haben wier nochvolgent weisthumb widerumb erneuwert, satzung und gerichtsordenung entschlossen und forther unsern underthanen und hindersassen schultheiss gericht und gantzer gemeinden zu Ellerstatt ubergeben, deren sich ein ieder inwohner doselbst gemess halten und gebrauchen soll, auch von kheiner partheien anders dann inhalt dernselben puncten $\mathrm{zu}$ handlen gestadtet werden. Und woh weiter darvon oder darzu gethann wurde, dasselbig soll nichtig, der richter den partheien desshalb inen aufgewanten costen abzulegen schuldig sein, und wöllen alle und iede vorgeiepte missbreuche an

1) Die Affenstein waren ein altes rheinländisches Adelsgeschlecht, dessen gleichnamiger Stammsitz bei Kreuznach lag. - ${ }^{2}$ ) Das kurpfälzische Hofgericht wurde ins Leben gerufen durch Friedrich den Siegreichen und war mit Gelehrten und mit Laien aus dem Ritterstande besetzt. Die Zeit der Gründung ist nicht ganz gewiss, doch war es nach der bei Büttinghausen, Beiträge zur Pfälz. Geschichte I, 100 abgedruckten Urkunde ohne Zweifel schon 1465 in Thätigkeit. Andrerseits bestand es wahrscheinlich 1452 noch nicht, da in der Ordnung, die Friedrich am 29. Mai dieses Jahres für die Universität gibt (Orig. der Heidelb. Univ.-Biblioth. Schrank II p. 4 Nr. 4), den Professoren in legibus et in canonibus die Theilnahme an demselben, wie es bald hernach geschah, nicht zur Pflicht gemacht wird. Die Ansicht Karlowa's in seiner Rede bei der akademischen Preisvertheilung, Heidelberg 1878, Note 24, wonach diese Gründung in besonderem Zusammenhang mit der Austreibung der Westfälischen Freigerichte, welche 1461 erfolgte, gestanden hat, dürfte die richtige sein. - ${ }^{3}$ ) Aus einem alten Adelsgeschlecht am Oberrhein, das die Ortsvogtei über das heutige Niederflörsheim in der Provinz Rheinhessen, Kreis Worms, hatte. 
unserm gericht zu Ellerstatt vormohls geiept worden, gantz und zumohl hiermit abgethann haben und diese nochvolgente ordnungen gehalten werden, doch uns und unsern erben vurbehaeltlich soliches zu minderen, zu mehren oder gar zu verandren und abzuthun, wie uns ieder zeit vur gut ansicht und gelieben mag.

Hie nachvolgt das weißthumb, so schultheiß und scheffen zu Ellerstatt zuh allen ongepotten dingdagen nun hinfurt weißen sollen.

In dem namen der allerhaeiligsten trinited, amen. Khundt und offenbare seihe allen und ieden, die dis nochvolgent geschrifft und weissthumb ihemer sehen, hơren oder selbs lesen, das in dem iar unsers haeils, als man schreibt thausent funfhundert funftzig und funf, auf sondag denn zwaeintzigsten monatsdag octobris zwischen ein und zwoh uuren noch mitten dag, in der Römer zinsszahl zu latin indictio genant XIII., bei hörschung und regierung dess allergrossmechtigsten, onuberwintlichsten, durchleuchtigsten hochgebornen fursten und herren herren Caroli de $\beta$ funften Römischen kaissers, zı allen zeiten mehrer $\mathbf{B}$. des reichs, khönig in Germanien, zu Castilien, Aragon, Leon, beider Sicilien, Hierusalem, Hungern, Dalmatien, Croatien, Navarra, Granaten, Toleten, Valentz, Galitien, Maiorica, Hispalis, Sardinien, Corduba, Corsica, Murcien, Gennis, Algarbien, Algezieren, Gibraltar, der Carnarischen und Indianischen inseln und der terre firmae, des occianischen möres etc., ertzhertzog zu Östereich, hertzog zu Burgundi, zu Lotringk, zu Brabant, zu Steir, zu Kernten, zu Krain, zu Limpurg, zu Lutzenburg, zu Geldren, zu Calabrien, zu Athen, zu Neopathrien und Wurtemberg etc., graff zu Habspurg, zu Flandern, zu Tirol, zu Gortz, zu Barcinon, zu Arthois, zu Burgund, pfaltzgrave zu Henigauw, zu Hohlant, zu Seehlant, zu Pfirt, zu Kiburg, zu Namur, zu Rossillien, zu Ceritania und zu Zütpfen, landgrave im Elsass, marggrave zu Burggauw, zu Oristani, zu Gociani und des heiligen Römischen rheichs furst zuh Schwaben, Cathalonia, Asturia etc., herr in Friesslant, auf der Windischen marck, zu Portenauw, zu Piscaia, zu Molin, zu Salins, zu Tripoli und zu Mecheln etc., unsers allergnedigsten herren, irrer kaeiserlichen maiestat regierung ime funf und dreissigsten und der andren reich aller ime viertzigsten, vor mir, hie nochbenanten auss bapstlicher undt Römischer kais. maiest. macht gewalt und begnadigung offenen notarien ${ }^{1}$ ) personlich gestanden und erschinen seindt die ersamen und erbarn schultheiss und scheffen des gerichts zu Ellerstatt in demselben dorf auf dem roth-

1) Ueber die Creirung der Notare von Päpsten und Kaisern vgl. Wetzell, System des ordentl. Civilprocesses. 3. Aufl. p. 235. - Im Namen des Kaisers übten sie vorzugsweise die Hofpfalzgrafen. So bekamen auch der Procancellar und der jeweilige Dekan der juristischen Facultät der Universität Heidelberg mit ihrer Ernennung zu kaiserlichen Pfalzgrafen, welche am 23. August 1745 durch den Kurfürsten Maximilian Josef von Baiern in seiner Eigenschaft als Reichsvicar erfolgte, dieses Recht. Orig. der Heidelb. Univ.-Biblioth. Schrank II p. $10 \mathrm{Nr} .3$. 
haus in der grosen stuben, mit namen Jost Anthis, schultheiss, Heinrich Weiss, Veltin Dietz, Lorentz Will, alle scheffen, und dann die gantz gemeinde gemeinigklichen doselbst. ${ }^{1}$ ) Und nochdem die gestrengen und edlen herren und ihunckhern, herr Wolff von Affenstein, ritter der churfurstlichen Pfalntz hoffrichter etc. und Friderich von Flerssheim der iunger mir bevelch in scluriften zugeschickt, irentwegen und als notarius puplicus nochvolgent weissthumb und gerichtsordnung inne irem dorf zu Ellerstatt vurgemelten schultheiss gericht und gantzer gemeinen, neben iren zugeordenten dhienern hienoch benant, offentlichen verlesen und alsdann instrumentieren solte, beneben und bei ir gerichtsbuch doselbst etc., welicher bevelch, so mir neben gemeltem weissthumb und gerichtsordnung zukhomen, laut von worten zu worten also.

Unsern freunllichen grues und alles guts zuvor. Lieber statschreiber, besonder gutter freundt. Nochdem vorn alters hero in unserm dörflin zu Ellerstatt iörlich zu dreien mohlen ein weissthumb gewesen, welches unsere vuraeltern aufgericht, aber dasselbige alters halben und ietzt der zeit onrichtig, so haben wier uns eines weissthumbs verglichen, wie dasselbig euch hiermit zukumpt. ${ }^{2}$ ) Hieruf ist

C. unser freuntlichs begeren und gesinnen, ir als notarius wöllent euch gon Ellerstatt verfugen, dieses weissthumb vur schultheiss und gericht, auch gemein offentlich verlesen und volgents instrumentieren, auch ins gerichtsbuch zu Ellerstatt schreiben und alles dasihenig vurnemen, das sich zu aufrichtung eines weissthumbs zu thun gepurth und wier selbs personlich zugegen wehren. Und dieweil wier eigner person selbsten zugegen nit erscheinen khunden, so wölent solchs bester gestalt und formb von unsernt wegen verrichten, wie ir euwers ampts zu thun wuol wissent. Hierin beschicht unser will und meinung, wie wier uns auch mit eigner hand underschriben, das wöllen wier gegen euch beschulden. Dat. Durmstein durstags noch Galli ${ }^{3}$ ) anno etc. LV.

Affenstein.

Friderich von Flerssheim der iunger m. pr.

\section{Subscriptio.}

Dem ernhaften Jacoben Waechtelin, stattschreibern zu Lambsheim unserm besondren gutten freundt.

1) Diese Form der Datirung etc. entspricht genau der Vorschrift, wie sie in \$ III der vom Kaiser Maximilian auf dem tage zu Köln am 8. October 1512 gegebenen Notariatsordnung festgesetzt worden war. Vgl. neue und vollständige Sammlung der Reichsabschiede. Frankf. 1747. II. Theil p. 151. - 2) So nimmt man z. B. auch in Embrach im Zürichschen mit Rücksicht auf die Veränderung der Zeiten im Jahr 1518 eine neue Aufzeichnung vor. Grimm p.111. - 3) 17. October. - Dirmstein 2 Stunden von Frankenthal gegen Worms zu gelegen; Lambsheim 1 Stunde von Frankenthal. 
Nach verlessung dis bevelchs habe ich inen sobald auch das bemelte mir mit ubersannte weissthumb auch vurgelesen, welliches noch seinem inhalt und von worten zu worten also laut.

Disses ist unßerer gnedigen herschaft Affensteins und Flerßhaeims als disßes dorfs Ellerstatt obergerichtsherren weisthumb undt recht in genantem dorf Ellerstatt und deren selben gemarcken, welches wier schulthaeiß und gerichtzscheffen, auch gantze gemeinde alle ior zuh dreien ongebottenen gerichtzdagen (. die man nent vollgericht.) bei unßern aeiden weißen, sprechen und erkhennen, wie dan von altem hero auf uns khomen. Namblich das erste auf den dinstag nach dem zwolften oder der heiligen dreier khunnig dag, das 2. auf dinstag nach der osterwochen und das dritte den dinstag nach sanct Johannes des heiligen thaufers dag, so fehr das dieselbige dinstag khaeine feirdage seien. ${ }^{1)}$ Welicher aber ein feierdag wehre, soll solches acht dag vurthan geruckt und alsdann wie vor bescheen sein solt, offentlich verkhundt werden.

Anfengklich weisen, sprechen und erkhennen wier die edlen gestrengen und ernvesten herren Wolffen von Affenstein ritter, der churfurstlichen Pfalntz hoffrichter, und Friderichen von Flerssheim den jungern dernselben zeit sie und alle ire erben und nochkhomenne vur unsere und des dorfs Ellerstatt rechte und obere gerichtsherren, soverr die gantze gemarcken ghedt, alle hohe und nidere obrigkheit, frevell und ongnad, gebott und verpott und sonst niemants anders, wie das von unsern aeltern und vorfahren vor onverdechtlichen ioren auf uns erwachsen. Wehr daruber sich dessen etwas underwunde, der dhete das mit gewalt und mit inbruch irer herligkheit.

D.

An solicher herligkheit hoher und niderer gefell, es seihe frevell, ongnad und allerlei bussen, wie die verwurckt, verbrochen oder gebuest werden mogen, erkhennen und weissen mir herren Wolfen von Affenstein und sein erben ein theil und Friderichen von Flerssheim und sein erben zwei theil.

Item weissen wier, das blutige wunden, druckene streich, gotzlesterer, uberstucken, uberzeunen, uberzackern, onbewisene scheltwort und dergleichen, verachtung der herren gebott, vergessenheit der pflichten etc. alles frevell seien; die hat obgenannte herschaft hoch oder nider und auch an dem leib zu straffen wie inen fuegt. Daran gepurt alweg Affenstein ein und Flerssheim zwen pfennig zu geben und zu nemmen. Also wann in malefitzsachen ein ubelthetige personn zu Ellerstatt durch die obrigkheit beclagt wurt an demselben costen zu gelten Affenstein ein und Flerssheim zwen pfennig zugerechnet.

Insgleichen weissen mir auch, so das hohe gericht zu bauwen und aufzurichten von nötten, das solches durch gemelter herschaft

1) Diese 3 Termine auch in dem älteren von Grimm publicirten Weisthum. 
costen wie vurgemelt, Affenstein ein und Flerssheim zwei theil erbauwen und dann durch die gantze gemeinde aufgericht werden soll. Alsdann mage gemelte unsere gnedig herschaft der gemeinden etlich fiertheil weins $\mathrm{zu}$ verdrincken schencken.

Von solicher herligkheit wegen weissen mir den beiden unsern gnedigen herrschaften alle ior zu Ellerstatt fallent an bed, namblich dreissig sechs malter korns Wormbsser moss auf Martini des heiligen bischofs dag, dessen Affenstein viertzehenhalb und Flerssheim zwentzig drithalb malder geburen. Darann geben die von Seebach sechtzehen malter und die von Schönveldt acht malder und sonst alle die in der gemarcken beguet seindt, geben die ubrigen zwelf malter. ${ }^{1}$ )

Item haben sie auch an gelt zu beden fahllen alle ior her Wolff aeilf schilling pfennig und drei pfennig so hat Flerssheim achthalb pfunt heller Speirer wehrung, das thut an Wormser oder gemeiner lantzwehrung funf th. neun albus drei pfennig. Solch bed, korn und gelt, ist ein ieder schultheiss zu Ellerstatt inzusamblen, der herschaft oder iren dhienern zu lufern und der gemeinden ire notturft zu werben alles auf ein meihl wegs weit schuldig, one der gemeinden schaden. Darumb hat er iedes viechs zwei stuck ledig ghen. ${ }^{1}$ )

Item weissen mir auch genanter herschaft auf einem ieden hauss zu Ellerstatt, so do bewohnet wurt, aussgescheiden die edelleut und

E. priesters heuser (so si von inen selbs bewohnet werden) ein fassnachthunh, daran Affenstein ein theil und Flerssheim zwei theil gepuren. ${ }^{1}$ )

Item wier weisen auch aller edelleut und der gaeistlichen gutter in der Ellerstatter gemarcken gelegen freih, wamn si durch ire eigenthumbsherren selbst gepaut werden, aber sonst gar nit. ${ }^{1}$ )

Item weissen wir auch viehIgenanter unser guedigen herschaft fronn und atz gemein oder geleich und ongemessen, also welcher under inen der fron oder atz bedarf und besucht durch sich oder seine dhiener, dem seind wier gehorsam zu leisten schuldig. ${ }^{1}$ )

Item wier wejssen auch, das genante herschaft in nötten das dorf Ellerstatt sampt dessen inwohner zu schutzen und zu schurmen schuldig seind mit hulf und beistand irer guten freundt, sovil mugklich. Und wess derenhalben vur costen aufgewandt wurdet, seind die clöster Seebach und Schönveld das halb und die gemeinde das ander halbig theil $\mathrm{zu}$ bezahlen schuldig. ${ }^{1}$ )

Item further weissen wier auch, woh sichs begebe, das ein argwenige ubelthaetige manus oder weibsperson ghon Ellerstatt kheme, dieselbige personn soll der schultheiss verwahrlich behalten. Woh dann ihemant kherne, und begerte die zu berechtigen, soll ime auf seinen costen gegunt werden. Woh aber niehmant kompt ienen zu beclagen,

1) Aus dem älteren Weisthum. 
mag soliche person alsdann mit vurwissen unser obrigkheit auf gepurlichen uhrfriden und nochdem er den aufgangenen costen bezahlt hat (so verr ers vermag, woh nit, sols die gemeinde bezahlen), wieder ledig gelossen werden.

Item weist man auch, ob iors drei oder mehre umb den schutz bitten, do mag die herschaft oder ire gesanten einen und die gemeind den andren erwehlen oder kiesen. Und die andren, so nit angenomen, sollent dernhalben von niemant geschmecht werden.

Weiter weist man einen gemeinen allmanngraben, der soll allweg auf rechts gebalten werden, der ghedt bei ihunckher Jörigen von Bachs scheuren an und ghadt durch den burgstaden durch das dorf und gerten, in dem dorf durch die wiesen und die winmorgen durch das bruckell in die böllengerten und kierssgerten, durch die khuewiessen in den Gennheimer ${ }^{1}$ ) schannengraben und dann wieder durch die khuewiessen durch die friessauwer und durch die bentzen und dann durch den sehe und wint an santbergen. Dieser graben soll offen onverspert sein, das iederman sein wasser zum nechsten on der gemein schaden darein kheren mag und soll unden auf dem boden funfthalb schuch und oben siebenthalben schuch weit sein.

Item weist man auch, das ein ieder muller, so zu Pfiffingen ${ }^{2}$ ) in der mullen wohnet, ist gezwungen, der gemeind zu Ellerstatt zu mahlen, F. desgleichen auch die gemeind widerumb zu ime auch gezwungen zu mahlen. Derowegen soll der muller alle wochen zwei mohl zum wenigsten ghon Ellerstatt fahren und den nachparn ire notturft mit dem mahlen thun.

Wann es khume, das ime einer zu mahlen geben hette, wenig oder vihl, und der muller brechte ime das sein nit volckhomen wieder, do soll derselbige einen, seinen nechsten nochparn hinder ime, und einen vur ime beruffen, die sollen ime das mehl messen; und finden si mangell, so mag dann er dem muhler den essell und sack oder karch und pferd nemmen, so lang und vil, bis er ime das sein wiederumb erstattet noch billichkheit. Und wann soliches geschee und dann derenselben nochparn einen oder ein andrer in die muhle kheme, so solle der muller sie des mit nichten entgelten lossen, sonder sie ehren und furdern vur allen andren, ime und seinem pferde essen und drincken geben, am fleischdag soll er ime das best thun und am fischdag zweierlei fisch und zweierlei wein geben, der nit saur noch faul ist. Wann auch die gemeinde zuh Ellerstat korn ausswendig kauft oder entlehnet, so ists bemelter muller drei meil wegs weit inen zu hohlen schuldig. Hat dann der gemein mann des ortz zehrung umbsonst, soll der muller dessen auch geniesen; mussen si aber ir gelt verzehren, so soll der muller sein irtten ${ }^{3}$ ) auch selbs gelten.

1) Gönheim 1 Stunde südöstlich von Dürkheim. - 2) 1/4 Stunde von Dürkheim. - $\left.{ }^{3}\right)$ Wirthsrechnung, Zeche. 
Were es aber sach, das einer oder mehr auss der gemeind zu Ellerstatt ander dann in gernelter muhlen zu Pfiffingen mahlen dethe oder thun wurde, woh in dann diser muller ergreiffen oder ereichen mag, soll er ime seinen muhlter nemen und in sein sack thun (so er anders einen bei ime hat); hat er aber kheinen sack bei ime, so mage er demselben seinen sack aussschutten und sein muhlter darein thun.

Item weist man das glockampt zu Ellerstatt der gemeinden doselbst zu verleilıen, und wann es ein pfarher begert, sols ime vur andren gegunt werden, so ferr er einen schuhler helt, der gelert seie ime zu altar dhienen und die glocken leuten khune. So es aber ein pfarrher nit begerte, so weist man es dem aeltisten kirchengeschwornen heim und also ihe einem noch dem andren. Und so dann iren kheiner solches annemmen wurde, es dann einem in der gemeinde geluhen, der darzu dugklich wehre und solches verburget, so sols doch mit wissen des pfarrhers geschehen.

Dieses alles wie erzehlt, weissen, sprechen und erkhennen wier schulthaeiss, gericht und gantze gemeinde zu Ellerstatt der obgenannten unserer gnedigen herschaft und dem dorf vur ein recht und gerechtigkheit, und ists ein ieder gemeinsmann und der do der herschaft gelopt und geschwohren hat, zu thun und zu halten schuldig bei demselben seinen aeid, auch alles, das'er wejss und erfure, das darwieder oder sonst rugbar wehre, er an den obgenanten dreien ongebottenen gerichtsdagen zu rugen und vurzupringen schuldig.

Darumb soll auch an bemelten dagen ein ieder gemeinsmann bei solchem verhör des weissthumbs sein bei peen und straff funftzehen G. pfennig, die seind der gemeinde, one sonderliche rechtmessige entschuldigung, leibs oder herren noit. Bei und zu solichem seind auch gezwungen die beide schaffner der clöster Sebach und Schönveldt, ${ }^{1}$ ) bei peen eines pfund hellers der gemeinden ir iedem, so er aussblib.

Es soll auch ein ieder schultheiss an bemelten dreien ongebottenen gerichtsdagen alsbald noch verhör des weissthumbs und vurbrachter rug alles eigentlich aufschreiben lossen, was rugbar und vurgebracht wurt, der herschaft das haben anzuzeigen, darmit sie das stroffen khunden und ir recht gehanthapt werde.

Wellicher aber etwas rugbar wiste und das nit anzeigt und verhielt oder verschwieg das und man wurde soliches von ime inen, denselben hetten dann die herschaft sonderlichen noch irem gefallen alles einem verrachter irs gebotts und uberdreter seines aeids zu straffen; darnoch wisse sich alle menigklich zu richten.

Noch solichem weissthumbs verlessen und der gemeinder gehapten bedacht habe ich nochbenanter notarius innen auch alsbald die gerichts-

1) Seebach altes Benediktinerinnenkloster, 1/2 Stunde südwestlich von Dürkheim; Schönfeld Benediktinerinnen- und später Cölestinerkonvent bei Dürkheim, da, wo jetzt die Saline sich befindet. 
ordnung mir neben dem weissthumb zukhomen, auf gehapten bevelch alles in beisein der benanten funfen mir zugeordenten Affensteins urid Flerssheimers dhienern auch vurgelessen von wort $\mathrm{zu}$ worten lautente wie volgt.

\section{Ordenung desß gerichts zu Ellerstatt.}

Unsere gnedige herren und junckhern, her Wolff von Affenstein ritter und Friederich von Flerssheim, auch ire erben und nochkhomene haben zu Ellerstatt zu allen zeiten einen schultheissen zu setzen und zu entsetzen, der das gericht in irem namen und von irentwegen behögen und obrigkheit, auch khein onformblich gericht oder sonst handlung daran geschehen fleissigs aufsehens habens, darzu alle gebott, verbott von der obrigkheit wegen thun soll.

Item so eines scheffens oder gerichtsmann noth sein wurde, soln den die andren scheffen etliche auss der gemeind zu Ellerstatt den gerichtsherren anzeigen, aus denen mag die obrigkheit oder, woh sie ein bessern wissen, ein andren auss der gemeind annemen und wehlen. Und welicher durch die obrigkheit also den scheffen geben wurt, derselbig soll mit dem nochvolgenten scheffenaeidt angenomen werden.

Item es soll das gericht mit siben scheffen, die in Ellerstatt gesessen und beguet, auch erhlicher gepurt und guten leumbdens sein, von der gerichtsobrigkheit gesetzt und mit nochvolgenten scheffenaeidt aufgenomen werden, die mit allem fleiss, sovil innen mugklich, aufmercken sollen, das formblich geclagt und der crieg bevestiget, auf das nit nichtigklichen gehandelt werde.

Item es soll auch in obgemeltem dorf Ellerstatt khein gericht noch dem mitdagimbs gehalten, sonder ieder zeit vur dem mitdagimbs gehandlet und geendet werden.

Item es soll auf ein ieden gerichtsdag schultheiss, scheffen und der gerichtsschreiber, beforab ongebotten und onverzuglich bei geschwornem aeid auf dem gewonlichen gerichtshauss oder platz, do das gericht ieder zeit gehalten wurdt, erscheinen und ir kheiner one ehhaft ursachen und erlauptnus des schultheissen aussbleiben, auch ir kheiner, die zeit das gericht wehret, one erlauptnus aufsteen oder abghen, sonder bis zu endt des gerichts ausswarten bei peen und straff eins weiss $\mathrm{H}$. pfennigs onnochlessig zu bezahlen, wellicher weisspfennig durch den schultheissen von einem iegklichen iedes mohl bei seinem aeid empfangen, in ein gemeine buchsen aufgehaben und zu gelegener zeit, so man ondas pflegt frölich zu sein, durch gemeine gerichtsgelieder verzert werden sollen, doch alles ieder zeit mit vergunstigung und vurwissen der obrigkheit. Woh aber einer oder mehr uber ein straff des weisspfennigs zum andren oder mer mohlen ausspleiben wurde und wieder mit eim albus sein ongehorsam zu bezahlen vermeint, so soll allweg, 
so oft und dick das bescheen, die straff allweg bis zum aussgang eins jeden iors gedoppelt und a ... ert ${ }^{1}$ ) werden, darmit der einitzige weisspfennig nit ein fahrlessigkeit bring und noch ordnung eins iors wieder noch der heiligen drei khunig dag mit einem angefangen und zu iedem gericht gemehrt werde.

Item dieweil nit alle wochen gericht $\mathrm{zu}$ halten verordenet, ist von onnöten geacht einem ieden zu seinem gefallen gericht zu verschaffen, sonder welicher das begert und nottdurftig ist, soll der nochbenanten gerichtsdag erwarten. Es wehre dann, das etwann ein frembde person ${ }^{2}$ ) oder ander ehhaften sachen, daran mergklichen gelegen und khein verlengerung oder aufzug leiden möchten, vurfahlent wehrent, alsdan mag man dem gericht zusamen gepietten bei gomelter straff auf die benante gerichtsdag darzu in allen notturftigen dingen die scheffen bei obgemelter peen gehorsam zu sein; darvon soll der, so das gericht zu versamblen begert, acht' albus erlegen, nemblich dem gericht vier, dem schultheissen zwen und dem gerichtsschreiber die andren zwen werden sollen.

Item demnoch das gericht zu Ellerstatt nit mehr dann mit siben scheffen besetzt, auch sonst gering und cleine handlungen $\mathrm{zu}$ erwarten haben, so haben wier ohgemelte gerichtsheren geordenet, das nun hinofuro alle ior drei ongebottene ding und gerichtsdag zu Ellerstatt gehalten werden sollen. Namblich das erste auf den dinstag noch dem zwelften oder der heiligen dreier khunig dag, das ander auf den dinstag noch der osterwochen, das dritt auf den dinstag noch sant Johannes des thaufers dag, doch woh ferien infallen, wurden alsdann solche termine acht dag vurgeruckt und dann den nachtvolgenten dag darnoch solch gemein oder offen gericht gehalten werden.

Item es sollen auch die scheffen sovihl inen mugklich, fleiss haben, das ein iede burgerliche sach, so vur innen in gericht hangt, in zweien ioren aufs lengst noch bevestigung des krieges zu end laufe. Sie sollen auch die partheien daruf zu handeln fleissig anhalten.

Item gebeut einer einem inwohner, cost ein heller und einem ausswohner zwen heller an das nechst gericht. Und so einer einem also zu gericht gebeut und der, dem vurgepotten wurt, auf das erst, ander noch dritte gebott nit erscheint, so soll der nit erscheinde darumb nit verdampt werden, sonder so er auf das ander, drit oder vierte gepot erscheinen wurde, soll er dem cleger sein rechtmessigen costen

I. auf messigung des gerichts zuvor aussrichten und bezahlen, ${ }^{3}$ ) von einem

1) Schwer leserliche Correctur. - 2) Das ältere Recht kannte manche Sonderstellung des Fremden; nicht überall war er benachtheiligt, bisweilen auch privilegirt. So hatte er z. B. Anspruch auf besonders schleunige Rechtshilfe, wenn er sich nur kurze Zeit an einem Orte aufhalten wollte (Gastgericht). Vgl. Stobbe, Handbuch des deutsch. Privatrechts I, 262. - ${ }^{3}$ ) Am Rande von derselben Hand lateinische 
jeden gebott, so er aussplihen ist, zehen pfennig geben, des soll der schultheiss zwen heller, das ander die scheffen haben. Alsdann soll in recht die partheien gehört werden und woh sich in recht gepurt, den antworter den crieg zu bevestigen, oder woh er sich solches zu thun weigerte, soll ime schub und bedacht, ob er absten oder sich in die criegsbevestigung inlossen wölle, bis zum nechsten gericht gegeben werden. Woh aber der antworter auf das vierte gebott auch nil erscheint, mag man zu insetzung ${ }^{1}$ ) der gutter, woh schon der crieg nit bevestigt, das ist zu latin ad immissionem bonorum ex primo decreto in recht furfahren, aber noch der criegshevestigung zu der haupthandlung furfahren. Es soll auch dem schultheisen vur ein iedes vurgebott und rechtsladung, es seie gleich umb ligens oder fahrens, zwen heller gehen werden. Und woh dann der beclagt soliche ligente guter darumb geclagt oder andre gutter, so sich in die beclagte hauptssuma erstrecken, bezitzet und inen hat, so soll derselbig bei hantgebener treuw gelossen werden, namblich das er dem rechten ausswarten wölle und hierzwischen die gutter nit verandren und in frembde hende veraeusern wölle. 2) Wann dann der beclagt nit besitzt oder inhat ligente gutter, so solle er geburliche burgschaft thun, woh er aber die auch nit bekhomen, uber das er sich dernhalben beworben und mag das mit seinem aeidt betheuren, so soll es zu dem aeidt, das ist juratoriam cautionem gelossen werden secundum textum glossam et doctores in lege: Qui iurato promisit.

Item wehr einen personlichen bekhumeren ${ }^{3}$ ) will, der soll dem schultheissen zwen pfennig und dem buttell zwen heller geben.

Item so ein aussmercker oder frembder einem inwohner zu Ellerstatt zu gericht gebeut umb ein schuld oder personlich clag, so soll der cleger dem inwohner burgen setzen vur den costen noch erkhanntnus des gerichts.

Dessgleichen soll der inwohner, so des ortz nit beguet ist mit ligenten guttern, dem frembden und heimischen burgschaft thun. Woh er aher nit burgen gehaben mag, soll er ein suma gelt, wie das gericht noch grosse und gestalt der sachen alle mohl messigung thut, hinder das gericht legen und dem schultheisen hantglupt thun an aeidts stat, woh es mehr costen wurde, das er dasselbig auch woll aussrichten, woh er dieselbigen gehaben mag, sonst bei der iuratorien cautionem gelossen wie oben gemelt. Woh aber der aussmercker oder frembde beguet wehre in Ellerstatter gemarcken, die gnungsann noch erkhantnus des gerichts vur den costen wehre, soll der cleger burgen zu setzen nit schuldig sein.

Verweisungen auf Bestimmungen des canonischen Rechts: c. 24 X 1, 29; c. $4 \times 2,14$ und c. 8 dist. 74 .

1) Beschlagnahme von Gerichts wegen. - ${ }^{2}$ ) Am Rande verwiesen auf 1. 15 D. II, 8 ; 1. 4 \$ 5 D. II, 11; 1. 2 D. II, 8; l. I, D. II, 8; 1. 25, D. L, 17. 3) verhaften.

Zeltschrift d. Savigny-Stiftung, IlI, Germ. Abth. 
Item woh einer ein clag auf ligente guter oder farente haben thut, soll man dem heclagten ein iede clag insunderheit verkhunden. So die auf ein inwohner geschicht, do soll durch den cleger dem buttel zwen heller, und so es auf ein aussmercker geschicht, von der meilen ein albus gegeben werden.

Item es sollen auch alle urtheil, so durch die scheffen gewisen, auf nochvolgente formb gesprochen werden, namblich: In dieser sachen zwischen $\mathrm{N}$. cleger an einem, und $\mathrm{N}$. antworter anderstheils, noch clag, antwort, verhörter kuntschaft, auch allem weiteren vurbringen und recht satz sprechen die scheffen zu recht, das etc.

K. Item es soll auch ein schreiber ${ }^{1}$ ) bei dem gericht sitzen und mit gelupten und nochgeschribenem aeidt aufgenomen werden und alles wes vurbracht wurdt gruntlich mercken, des achtung haben und wie ime vurbracht wurt aufschreiben und nit anders bei seinem geschwornen aeidt.

Item es sollen auch ihe von sechs ziehlen auf bogen, die nit zu lang oder zu gross, sonder mit zimblichen buchstaben geschriben seindt, die vurbringer dem schreiber ein pfenig zu geben schuldig sein.

Item darmit die scheffen irer arbeit und versaumnus etlicher mossen erstattung empfahen, so soll von iegklicher endt urtheil, eh und zuvor dasselb eröffenet, von beiden theilen drei schilling heller, iede parthei neun pfennig, dargelegt werden. Darvon soll haben der schreiber zwen pfennig und das ubrig den scheffen pleiben.

Von einem beiurtheil ${ }^{2}$ ) zwölf pfennig, soll auch iede parthei sechs pfennig vor eröffenung der urtheil dargelegt und darvon dem schreiber zwen pfenig, das ander den scheffen werden.

Item ein bote cost sechs heller, soll werden dem schreiber zwen, drei den scheffen und einer dem schultheissen. Wann mans mit gericht thut, soh costs vier schilling dem gericht und drei pfennig dem schultheisen.

Item erbschaft, einkhintschaft, rachtung, verdraeg, heurat etc. und dergleichen handlungen in das gerichtsbuch zu schreiben kost vier schilling dem gericht, dem schultheissen vier pfenning, und dem schreiber noch gelegenheit der sachen.

Item ein satzung ${ }^{3}$ ) cost ein fierteil weins, und vur ieglichen morgen velds und hauss soll der verlieher eim schreiber ein pfennig geben von solichem in das gerichtsbuch zu schreiben.

1) Der Gerichtsschreiber kommt als verpflichteter Beamte in der älteren deutschen Gerichtsverfassung nicht vor, fand aber nach und nach, besonders seitdem die Reichsgesetzgebung solche in das Reichskammergericht eingeführt hatte, mehr und mehr Eingang. Wetzell p. $427 \mathrm{ff}$. - 2) Ueber diese Beiurtheile oder Zwischenbescheide, die eine vorgängige causae cognitio voraussetzen, vgl. Wetzell p. 652 ff. 3) Pfändung. 
Item so ein gut ein mohl vur gericht verlegt wurt, so soll das gericht $\mathrm{zu}$ kheiner weitern verlegung ghen, darein das gut verlegt werden wolt, das gut seie daun zuvor von der ersten verlegung wieder erledigt.

Item das gerichtsbuch zu lessen cost dem gericht ein halpviertheil weins und iede parthei dem schreiber vier pfennig. Wann es aber ein parthei allein lessen lost, soll si den albus auch allein geben.

Item ein aufhohlung cost dem gericht vier schilling und den schultheissen drei pfennig.

\section{Den abdrieb, ${ }^{1}$ ) wie es darmit gehalten werden soll etc.}

Ein erb dreipt den kaufer in ligenten gutteren ab in ior und dag, und soll der kaufer das gutt in obgenanter zeit nit schedigen, sonder sich allein der abnutzung gebrauchen und soll der erb vur solich gut schwehren, das er die lossung ime und niemants anders thue. So soll der erst kaufer und verkaufer, die den kauf und verkauf gethann handt, auch schwehren, das solicher kauf und verkauf umb die suma und dermossen als si sagen gescheen seie, getreuwlich und one alle geverdt. Gleicher gestalt soll dem erben zu wechsslen, kaufen, dauschen, bezahlung geben oder dergleichen die lossung auch vorbehalten werden.

Item in allen sachen, so vur den scheffen rechtlich gehandelt und zu urtheil beschlosßen, deren sie zu sprechen nit verstendig werden, $L$. mögen die scheffen von beiden partheien, einen sovil als von der andren gelt noch gestalt und gröse der sachen nemen und alsdann die acta solicher sachen aller mossen, wie die vur innen erlaut haben, schriftlich abcoppieren und mit zweien scheffen der obrigkheit ubersenden. Dieselben sollen furter die acta besichtigen und alsdann, was sie ferner von innen bescheiden, demselben noch irem besten verstenntnus urtheilen. Und so an solichem gelegten gelt uberblibe, dasselbig den partheien wieder lossen, woh aber gepreste, das sollen ehe und zuvor der eröffnung der urtheil die partheien den scheffen darlegen und bezahlen.

Und so das urtheil gefast ist, sollen die scheffen beiden partheien furderlichen die zu empfahen dag ansetzen, die urtheil geben und eröffnen.

Item so von einem urtheil appelliert wurt, solliche appellationn soll niergent andershin gethan werden dann vur beide gerichtsherren Affenstein und Flerssheim und dem, so appelliert, auch seinem gegen-

1) Ueber den Retrakt, auch Einspruchsrecht, Abtrieb, Nähergeltung etc. genannt, vgl. Stobbe II, $120 \mathrm{ff}$. Während sonst beim Tausch kein Retrakt stattfindet, scheint er in den Gegenden, denen unser Weisthum angehört, zulässig gewesen zu sein; wenigstens erlaubt ihn auch das Wormser Statut (Stobbe pag. 133 Note 16). 
Miscellen.

theil auf ir begern appostolos ${ }^{1}$ ) gepurlicher moss under dem gerichtsingesigell gegeben werden. Dargegen soll iedes theil, so die acta haben will, dem gericht drei albus und dem schreiber sein zimbliche besoldung noch gestalt der sachen geben.

Hienach volgen spartelnsachen, das seindt die, so sie

in gericht vurbracht werden, darvon genomen und

gegeben etc.

Item die erst burgerliche clag, so einer am gericht thut, soll der vurbringer geben zwelf pfenning, deren sollen den scheffen zehen pfenning, dem schultheisen zwen heller und dem schreiber ein heller gegeben werden. Verbotz der mann, so cost es noch sechs heller, drei dem gericht, ein dem schultheisen und zwen dem schreiber.

Item die ander clag uber viertzehen dag hernach kost sechs heller, drei den scheffen, ein dem schultheissen und zwen heller dem schreiber. Desgleichen soll es mit der dritten clag auch gehalten werden.

Item so einer ein peinliche ansprach on gericht vurbringt, soll er von der ersten clag zwen albus geben, des sollen den scheffen werden zehen pfennig, dem schultheissen vier pfennig und dem schreiber zwen pfennig werden.

Item die ander peinlich clag oder ansproch uber vierzehen dag darnoch cost ein albus, dem gericht vier pfennig, dem schultheisen vier heller und dem schreiber zwen heller. Desgleichen soll es mit der dritten clay auch gehalten werden. Und soliche peinliche clag soll der schultheiss von der gerichtsherren als der obrigkheit wegen verpotten ${ }^{2}$ ) und die partheien annemen. Welicher theil dernhalben in recht verlustigt wurt, der soll (sover sich die peinlich bandlung in der Ellerstatter gerichts gepiet und gemarcken begeben hat) soliches gegen den gerichtsherren und obrigkheit abdragen.

Item ein ieder cleger soll seine drei clagen auf die nechsten gerichtsdag nocheinander, auch den dag der inwehrung, wie im durch den schultheissen benent wurt, volfuren und daruf handlen, wie sich gepurt. Und versaumpt der clegere solicher clagen eine onne leibs oder herren noit, so soll er seine gethanne clagen alle verluhren haben, und so er vorther in gerichte handlen will, soll er seine clagen von neuwem thun.

M. Item woh ein gebot auf einen feirdag gefiehle, auf den dag daruf einer ein clag thun sollte, so soll der cleger die clag auf den nechsten werckdag darnoch thun in mossen auf den feierdag darvir gescheen sein sollt.

1) Der Unterrichter war verpflichtet dem Appellanten einen Bericht, in welchem er die Sache an den Oberrichter dimittirt, deshalb literae dimissoriae oder apostoli genannt, auszuhändigen. Vgl. Wetzell p. 726. 2) vorladen. 
Item welicher do will auf des gegentheils vurbringen, es geschee schriftlich oder muntlich, abschrift und schub begert, soll ime auf sein billichen costen zugelossen bis auf den nechsten gerichtsdag gesetzt werden. Deshalben soll er in das gericht sechs heller geben, des geburt dem gericht drei heller, dem schultheissen ein, dem schreiber zwen und sollen hiermit schultheiss und scheffen aufmerckung haben, das gevorliche und onnotturftige ufschub nit gestaltet werden.

Volgt hernach iuramentum calumniae, das ist der aeidt vor geverde etc.

\section{Der cleger soll allßo schweren.}

Ich glaub und weiss anders nit, dann das mein krieg und sach recht und war seie. Und was ich ine der sachen gefragt und mir vurgehalten wurt, die wahrheit deshalb nit verhalten, khein schenck oder gab, dardurch das gericht verzogen oder verkhert werden mochte, geben habe, oder zukunftigklich geben will, auch khein geferlichen verzug in der sachen geprauchen, sonder die warheit vurbringen will, auch kheiner valschen beweissung oder gezeugnuss mich gebrauchen, so wohr mir gott helfe der allmechtige.

\section{Der antworter solf allßo schweren.}

Ich glaub und weiss anders nit, dann das ich ein gute sach hab, will auch ein warhaftige antwort geben, und was ich in der sachen gefragt werde dem rechten zu guttem meins wissens die warheit bekhennen, khein geferlichen aufschub oder ausszug die sachen aufzuhalten durch mich selbs oder in rhotschlegen ansuchen, khein schaenck geben noch nehmen lossen will, dardurch das recht verzogen oder mir zu steuwr verkhoert möcht werden, auch mich keiner falschen beweissung gebrauchen, als mir gott helf der allmechtige.

\section{Der aeidt vor die armutt.}

Duh solt geloben und ein aeid zu gott dem almechtigen schwehren, das du so arm seiest und an ligenter und fahrenter hab, auch nit an schuldigen, sovil habest noch vermögest, das du weist den richter, schreiber, rotgeber, redener, pittell oder andren personnen etc. umb ir arbeit, brief und anders zu volfurung und notturftigkheit deines rechtens, so dir zu geben geburt und schuldig wurst, nit bezahlen noch bekhommen magst, das duh auch dein hab gut oder schulden nit verauserst, verpfenndest, versetzest, verschenckest, hiriderhaltest oder ihemant zugestelt oder ubergeben habest, und so du mit recht dein sach behalten oder sonst zı besserem vermögen und reichtumb khomen wurdest, das du alsdann einen ieden obgenanten angezeigten noch seiner gebur und verdienst bezahlung, vergnugung und aussrachtung thun wölest, alles getreuwlich und ongevorlichen. 
Item so einem ein aeidt zu bewehrung seines rechtens mit recht aufgelegt wurdt, dem soll der richter ein zeit noch gestalt der sachen geben, in derselben zeit soll der, dem der aeidt aufgelegt wurt, den aeidt thun.

N. Item wer ein erkanntnus vur dem schultheisen und scheffen thutt, soll sechs heller geben, darvon geburt dem schultheissen einer, den scheffen drei und dem schreiber zwen heller. Beschicht aber ein erkantnus allein vur dem schultheissen, so mage der schultheiss die obgenanten sechs heller allein behalten, er wölte dann, das die erkantnus solt ins gerichtsbuch geschriben werden, so soll es getheilt werden wie obstett. Und so einer ein schuld schuldig und gestendig ist und doch nit bezahlen will, so mag der glaubiger den schuldener vur den schultheissen forderen und obe er nit vurkhomen wolt, so dann der glaubiger dem schultheissen zwen heller geit, so soll der schultheiss alsdann dem schuldner vur sich gebietten, und so der schuldener der schulden vur dem schultheissen auch gestedt, soll er im vierzehen dag zielle machen, den glaubiger za bezahlen. Und so der schuldener in der zeit nit bezahlte, soll der schultheiss dem glaubiger auf sein gesinnen gepurlich pfand durch den puttell in mossen wie vor von den verpfennten guttern geschriben stedt, geben und volgen lossen. Ist aber die erkhannte schuld liedlonn ${ }^{1}$ ) und will der, dem man die schuldig ist, nit mit willen ziehl geben, so soll ime auf stund gepurlich pfandt gegeben werden.

Item so ihemant wehre, der das gerichtsbuch bedurfen wurde, so es nit gerichtsdag wehre, der oder die sollen ann schultheisen begeren, die scheffen zu versamblen, alsdann soll der schultheiss den buttell bescheiden die scheffen zu verpotten zusamen zu khomen. Des soll der, der sie versamblen lost, dem buttell vier pfennig und dem gericht vier schilling geben, woh aber der oder die etwas verners furbringen oder in gerichtshendlen offnen thette, der sollt darvon geben, wie gesetzt ist.

Item ein aufgab, die ame gericht oder sonst vurh schultheisen und scheffen geschicht, cost zwelf pfennig, zehen pfennig dem gericht und zwen pfennig dem schultheissen. Verbots der mann, so costs noch sechs heller, geburen drei den scheffen, einer dem schultheissen und zwen dem schreiber. Und von einem ieden morgen velds oder haus, so aufgeben wurt, soll der aufgeber dem schreiber ein pfennig geben, der auch soliches alsbald das ior und den dag, daruf das geschehen ist, in das gerichtsbuch schreiben soll.

Item so einer zugelosßen wurt khuntschaft zu fueren, so soll der zeugenfuerer die articcell, daruf die zeugen verhört werden sollen, auch die personen und namen der zeigen dem wiedertheil schriftlich zuzuschicken beschaeiden werden, auch den dag verkhunden mit dem puttell, daruf die zeigen verhört werden sollen, ob der widertheil wölle, die

1) Verdienter Lohn. 
zeugen sehen, globen und schwehren und fragstuck auf die gegebenen articcull inlegen, das hat er macht. Die scheffen sollen auch in beisein des gerichtsschreibers die zeugen mit glupten und aeiden, wie nochstedt, vur irer sag aufnemen und alsdann ein iegklichen insunderheit auf artickel und fragstuck eigentlich verhören und desselbigen sag aufschreiben lossen, auch den zeugen bei dem aeid gepietten, seiner sag halben niemantz nichts za eroffnen.

Es soll auch durch den zeugenfuerer von einem iegklichen artickell, so dick ein zeug daruf verhört, zwen pfennig gegeben werden, das gehört halb dem schreiber, das ander halbtheil gehört den gerichtsscheffen.

So ein parthei die verhör der gezeugen in gericht offen lesseu 0 . lost, so soll die von einem iedem platt zwen pfennig geben dem schreiber. Weliche parthei aber die nit verlessen lost, sonder deren ein abschrift begert, soll ime vor messige belohnung des schreibers gegeben werden.

Welicher also zugelossen wurt zeugen $\mathrm{zu}$ fueren ${ }^{1}$ ) und seine gezeugen, so er bedarf, gebrauchen ist, der soll einem ieden zeugen sein geburlich daglonn und zimbliche zehrung geben, dieweil derselbige zeugg der sachen ein furderung geben hat.

Wann einer einen andren vur dem gericht monpar oder mechtig macht, das cost zwentzig sechs heller, darvon dem schreiber zwen heller die monparschaft einzuschreiben, vier heller dem schultheissen und zwentzig heller den scheffen geburen soll.

Wer einen vursprechen vur gericht in burgerlichen sachen braucht, und so die sach under zwentzig gulden ist, zwen albus vursprechen lonh, und so die sach uber zwentzig gulden ist, drei albus zu vursprechen lonh vur gerichtscosten zu schetzen oder taxieren.

Wer einen mit recht erlangt ihemant zu pfenden oder sonst pfant vergunt seind, der soll dem schultheisen zehen pfennig und dem buttell zwen pfennig geben, so soll der schultheiss den buttell leihen die pfandung zu thun, und soll es mit der pfandung wie von alter her gehalten werden, und so der gebuttell auf rindt oder ander gepfant viech wartet, soll im zimbliche belohnung werden noch gestalt der sachen.

Welicher inwohner des gerichts sigil bedarf und darumb bittet, das cost neun pfennig; des gepurt dem schultheissen einer und die andren achte den gerichtsscheffen.

Item so ihemant ghon Ellerstatt ziehen wolt, der soll brief und sigil von seiner obrigkheit oder dem gericht bringen darhinder er gesessen ist, das er mit wissen und mit willen von inen gezogen seihe, und das er dem schultheissen zu Ellerstatt zusage, das er khein noch-

1) D. h. wer zum Beweise durch Zeugen kommt. 
Miscellen.

volgenten herren oder zanck habe. Woh er aber etwas hader oder zancks hetle, soll er den dem schultheisen bei seinem aeidt sagen, wie er stehe. Alsdann soll der scbultheiss der obrigkheit soliches anbringen und daruber bescheid nemen, ob er den mit irrung soll innemen oder nit. Und so der ingenomen wurt, soll er der obrigkheit ein halben gulden und der gemeind zu Ellerstatt auch ein halben gulden geben, er hab irrung oder nit.

Item so einer ein anleidt begert, so soll die gemein gantz auf einen dag, den der schultheiss darzu benennen wurt, ghen und iunge knaben, die uber siben ior seindt, mit innen nemen und das angeleidt weissen, und sollen die alten, die der angeleit wissens haben, vurghen. Welichem theil dann etwas abgewissen wurt, der solle der gemeinden alsbald vier schilling heller geben.

Item so zwenn mit weingarten neben einander geforchen, soll die forch zwischen inen dreier werckschuch weit sein und ieder anderhalben schuch von dem stein oder der fuorch setzen.

Item welicher ein mohlstein mit ongeverdt ausszackert, aussgrub

P. oder verstopft oder wie er den veraendert, der soll, so er besagt wurt, zehen schilling geben, halbert der herschaft und halb der gemeinden und den stein auf seinen costen wieder setzen lossen. Erfint sich aber, das er soliches mit geverd gethann hette, oder einer thun wurde, der soll der gemeind zehen schilling geben und further in der obrigkheit straff sten. Darumb soll ein ieder gewarnet sein, das er seinem knecht und gesinde bevehle, der stein gut acht zu haben, dann man wurt den maeister darumb straffen.

Hie nachvolgen die aeidt einer iegklichen personen, als schulthaeißen, scheffen, gerichtsschreiber, buttell, messer und der gemeins leuth.

\section{Volgt erstlichs desß schulthaeißen aeidt.}

Ein schultheiss soll der obrigkheit und gerichtsherren mit handtgebenen treuwen geloben und mit aufgeregten fingern ein aeidt zu got dem allmechtigen schwehren, den gerichtsherren gewertig, getreuw und holt zu sein, iren nutz und fromen und kheinen schaden thun noch schaffen gethan werden, des gerichts heimlichkheit verschweigen, auch den scheffen helfen getreuwlich schutzen den armen als den reichen und die urtheil oder scheffen in gerichtsgescheften und sonst in andren zimblichen fehlen, auch die gantze gemeinde von der gerichts und oberherren wegen beschirmen, menigklichen zu recht behulflich sein, dem armen gleich dem reichen geburlichs vurnemen hanthaben und sich dagegen nichs bewegen lossen, auch von den partheien oder ihemants anders umb kheinerlei sachen wegen, so im gericht hangt oder hangen wurt, kheine gaben, schencken oder einiche nutzung durch sich 
selbs oder andere, wie das menschen sin erdencken möchte, nemen oder zu nemen zulosßen, auch die sachen bösser meinung nit aufhalten oder verziehen, weder umb freundtschaft noch feindtschaft willen in kheinen weg und one alle geverde.

\section{Der scheffen aeidt.}

Ein iegklicher, der zu einem gerichtsscheffen aufgenomen wurt, der soll den gerichtsheren und dem schultheissen von der gerichtsherren wegen geloben und ein aeidt leiblich zu gott dem allmechtigen thun, den gerichtsherren, schultheiss unde gericht getreuwlich und mit fleiss ob zu sein, in sachen, so vur inen bracht wurt, dem hohen und dem nidern, dem armen als dem reichen, noch seinem besten verstand gleich zu richten und mit nichten sich etwas dargegen bewegen lossen, auch von den partheien oder iemants anders khaeiner sachen halben, so in gericht hangt oder anhenig gemacht wurt, kheine gab, schenck oder einiche nutzung durch sich selbst oder andere, wie das erdacht werden möchte, zu nemen oder nemen lossen, auch kein sonder parthei oder anhang im gericht machen, noch ime seiner urtheil zu fallent suchen und khaeiner parthei rotten oder wahrnen, und was in ratschlaegen und sachen gehandelt wurt, den partheien oder niemantz zu eröffıen, vur oder noch der urtheil, auch die sachen arger meinung mit nichten aufhalten oder verziehen, darzu der herschaft und der gemeinden ire gerechtigkheit zu allen ongebotten gerichtsdagen getreuwlich weissen laut des gerichtsbuchs, aller dingen ongeverdt.

\section{Desß gerichtzschreibers aidt.}

Der gerichtsschreiber soll dem schultheissen geloben und einen Q. leiblichen aeid zu gott dem allmechtigen schwehren, seinem ampt getreuwlich ob zu sein mit aufschreiben, lessen und andrem auch die brieff und alles, was in gericht bracht wurt, getreuwlichen zu bewahren, und den partheien oder niemantz anders zu eröffnen, was in denen sachen von rotschlegen des schultheisen und scheffen gehandelt wurt, auch die heimlichen gerichtshendell niemantz zu eröffnen, lessen oder sehen lossen und khein copei von den inbrachten briefen, acta und schriften den partheien noch niemant anders geben, one erlaub oder erkantrus der scheffen, auch kheiner partheien wieder die ander, etwas rotten noch wahrnen, noch thein schenck nemen noch ime zu nutz nemen lossen in kheinen weg, wie das möchte erdacht werden one alle geverde.

\section{Desß buttells aeidt.}

So ein buttell von nötten wurdt, sollen die scheffen der gerichtsobrigkheit etliche auss der gemeinden zu Ellerstatt, die sie vermeinen darzu duglich und dienlich zu sein, anzeigen, und ob die obrigkheit alsdann einen bessern, dann angezeigt, wuste, welicher dann also auss der gemeinden der obrigkheit angenem wehre, der soll ein buttell sein 
und geben, und soll alsdann mit aufgehabenen fingern ein aeidt zu gott dem almechtigen schwehren, den gerichtsherren, schultheiss und dem gericht getreuw und hohlt zu sein, iren schaden wahrnen, kheinen thun noch schaffen gethan werden, seinem ampt getreuwlich vor sein und was ime durch den schultheissen oder die scheffen bevohlen wurt, sein botschaft fleisig werben, in mossen ime bevohlen wurt, one einiche aussflucht oder verlengherlichen ausszug, und wess er durch den schultheissen oder die scheffen gefragt wurt, seiner amptzgescheften oder handlungen die warheit bekhennen und sich dagegen nichs bewegen lossen, das die warheit verhindern möchte und darn die onwarheit iren vurgang haben wurde, wes er auch von dem schultheissen und scheffen haeimlichs sehen, hören oder vernemen wurde, dasselhig ewigklichen verschweigen, alles getreuwlich und ongevorlichen.

\section{Der mesßer aeidt.}

Ein iegklicher messer soll mit handgebenen treuwen den gerichtsherren oder iren schultheissen als an statt der obrigkheit geloben und further mit aufgehabenen fingern ein aeid nochvolgenter mossen zu gott dem allmechtigen schwehren, namblich also. So oft und dick ich mit andren meinen gesellen in geburlicher zeit zu messen geheissen oder durch den schultheisen von der obrigkheit wegen mossung zu thun bescheiden wurd, gehorsam sein will und mit der rutten, sovil mich mein gewissen und verstand weissen mag, eins iegklichen gerechtigkheit ersuchen und was sich gepurt, und nit weiter, abtheilen und mit stein setzen, beschliessen auch khein onrechten stein, so ich den erfinden wurde, wieder setzen, die gerechtigkheit sei dann zuvor mit wissen und mit der rutten wiederumb ersucht worden, auch die heimlichkheit der stein und sonst mit rhotschlegen und allen handlungen, die sich in messerampten begeben und erhalten gewertig sein, auch mich dargegen nichs bewegen lossen forcht, feindschaft, gunst, gnad, noch giftung oder gaben mit nichten nemen, noch mir zu nutz nemen lossen, inne kheinen

R. weg, wie das immer möcht erdacht werden, dardurch die gerechtigkheit verhindert werden möchte, alles getreuwlich und ongevorlichen.

Ein ieder, so in die gemeinde zuh Ellerstatt begert und eingelosßen wurdt, soll mit volgentem aeide auf und angenomen werden.

Ein ieder gemeinsmann soll dem schultheisen von der obrigkheit wegen mit hantgebenen treuwen und aufgehabenen fingern ein aeidt zu gott dem allmechtigen schweren, den gerichtsherren, schultheisen, gericht und gantzer gemeinden getreuw und holdt zu sein, die frue und spoid vur irem schaden wahrnen, dessen kheinen thun noch schaffen gethann werden, freiheit und herligkheit iors $\mathrm{zu}$ ongebottenen gerichtsdagen der obrigkheit gerichtsherren und gemeind vur recht geweist und von alters herkhomen seind, getreuwlich helfen hanthaben, auch kheinen inwohner mit ausslendigem oder frembden rechten furnemen, 
umb deren sachen er ietzt zu thun hette, oder nochmohls zu thun gewinnen wurde, zu Ellerstatt und der obrigkheit desselben gerichts (sover im rechts verholfen wurt) benungen lossen, alles getreuwlich und sonder geverden oder argelist, so wohr ime gott helfe der allmechtige.

Nota. Dieße erzehlte aeidt liest man den perßonen, so sie zu gemelten aemptern oder in gemeine angenomen und erwöhlet seindt, vurh und volgentz sollen sie dieße nachgheende wort nochsagen mit aufgeregten fingern also lautente.

Als mir vurgeleßen ist inhalt aller solichen claußullen, puncten und articcell, wille ich samptlich und sonderlich beih meiner gethonen glupt und aeidt getreulichen nochkhomen, alles on geverde und argelist, so wahr mir gott belfe und sein haeiliges laeiden, amen.

Erinnerung der peen und straff maeinaeidts, falscher gezeugnus, welche ermanung der richter onbegert einicher partheien vor dem aufnem oder verhör den zeugen vurhalten soll.

Ein falscher maeinaeidiger gezeug erlangt diese nochvolgente straffen. Zum ersten: peen und straff seiner sehlen, soverr er demselbigen aeidt nit genung thut, in welichem aeidt er das leiden unsers lieben herren Jhesu Christi beleidiget, so er onrecht sagen wurt. Soliche straff und peen wehret immer und ewiglichen.

Zum anderen: so ein zeug ein falsche kuntschaft gebe und das offenbar wurde, so wehr derselbige zeug seiner obrigkheit mit leib und leben in straff verfallen und ist wurdig, das im sein falsche zung hinden zum nacken aussgerissen werde.

Zum dritten soll man ein meinaeidigen gezeugen den dhaumen sampt den nechsten zweien fingern an der rechten handt abhauwen, mit welchen er den aeidt bezeugt und faelschlich geiebt hat, dann in $\mathbf{S}$. aufregckung gemelter dreier finger ein ieder die allerhaeiligste dreifaltigkhaeit bedeutet und zu wurckung solches seines aeidts erfordert und durch den falschen zeugen nit allein in den haeiligen geist sundiget, welche sunden dann nimmermehr aussgelöscht werden noch laut und inhalt heiliger göttlicher geschrifft, sonder er sundiget in gott den vatter, in gott den sohn und in gott den haeiligen gaeist auch in dem himmell, welche sunden ewigklichen nit verzigen werden.

Zum lesten bringt ein falscher gezeug nit allein sich in soliche ewige und zeitliche peen und straff, sonder bedreugt den richter und gibt ime ursach ein falsch und onrecht urtheil zu sprechen, dardurch dem rechthabenten onrechts begegnet und seiner sachen wider recht und billigh entsetzt und einem andren ingesetzt wurt, dernhalb der falsch gezeug schuldig dem verlüstigen seiner sagen halben volkhomene erstattung $\mathrm{zu}$ thun, der hauptsachen und alles costens. 
Volgt nun der aeidt, mit welchem ein ieder gezeug auf und angenomen werden soll.

Ein iegklicher gezeug (so zu zeigen zugelossen wurt) der soll mit hantgebenen treuwen geloben und dann mit aufgehabenen fingern ein aeid zu gott dem allmechtigen schwehren bei der vurgelesßen peen und straff auf articcell und fragstuck, und sovil im vurgelesen und er gefragt wurt, wess im desselben wissens, dem rechten zu steuwer die lauter warheit zu sagen, auch sich dargegen nichs bewegen noch hinderen losßen forcht, gunst, gaben, freuntschaft oder feindschaft noch etwas anders umb nutz oder schadens willen, dessen alles er in solichem vermeiden und nit nernen, thun noch schaffen oder gestatten zu gescheen in khaeinen weg, wie das namen gehahen khan oder mag, nichs aussgenomen, darmit nit die onwarheit gefurdert und die warheit gehindert werde, alles getreuwlich, ongevorlich und sonder arglistigkheit, soh wahr ime gott helfe der allmechtig.

Nota. Wann nun die erinnerung, straff und peen maeinaeidts, auch die erzelte aeidt den zeigen ingebildet und vorgeleßen wurdt, so soll alsdann ein ieder zeig seine drei fordersten finger an der rechten hant vorgenant aufrecken und dem verhörer also nachsprechen.

Dem vurgeleßen aeidt bei gehörte peen und straff maeinaeidts ermanung mitsampt allen puncten und articcelen darin begriffen, will ich also gemeinlich und sonderlichen nochkhomen, wie sichs einem fromen gezeugen gezimpt und gepurt, alles getreuwlich sonder gevor und alle argelist, so wahr mir gott helfe und sein bitters leiden. Amen.

Nachvolgente gemeine fragstuck, so dem zeugenverhörer zu wisßen geburen, soll auB rechtlichem ampt durch inen zuvor den articclen vurgehalten, ire antwort drauf gehoert und dann aeigentlich beschriben werden.

Item was stands, alters, reichthumbs, woh er burtig, mit hauss gesessen und wie lang er do gewohnt, ob er auch in kaiserlichen bann T. oder acht, noch einicher partheien mit pflicht, glupt, aeiden verbunden oder sonst mit sipschaft verwandt, und wie nohet die freundschaft seihe.

Item ob er ihemahls etwas in dieser sachen gerotthen und welcher partheien er das gethann, oder sich selbs noch andre zu zeigen angeben mit denen sich beredt habe, waß er oder si sagen sollen oder wöllen.

Item ob er auch vurhin ersucht, ermanet, gebetten, gelernet, was und wie er sagen soll und von wemb solches geschehen, oder ob er auch einicher partheien dieser sachen zu sigen gunstiger dann der andren und welichern er also gunstig, ob im auch etwas gegeben, geschenckt, verheissen, getrauwet und er dernhalben nutz oder schadens sich hoffen oder besorgen und von wemb er solichs gewertig seihe oder schon empfangen habe. 
Item es soll auch einem ieden zeugen angezeigt und des ermanet werden, das seine khuntschaft sag dem gegentheil eröffnet und gestattet werden darwider zu reden, und so alsdann in seiner sag und kuntschaft einicher falsch und onwarheit befunden wurt, er zeug deshalb angeclagt und in vorbestimpter mossen ongestrafft nit bleibe.

Es soll auch ein ieder zeig underricht und gewahrnet werden, das nit allein der vur ein falschen gezeugen geacht wurt, der die onwarheit sagt, sonder auch der, so die warheit weiss und si verschweigt und nit sagt.

Weiter soll auch ein ieder gezeug sonderlich und eigentlich befragt werden auf ein ieden articcell ob er wisse den artickul wahr sein und so er den wahr sein sagt, soll er befragt werden die ursach seines wissens. Sagt er aber den articcell nit wahr sein oder des kheins wissers haben, soll er darbei gelossen und derenhalben nit ferner gefragt werden.

Darnach soll ein ieder zeug auf die artickell und sondere fragstuck von den partheien ingelegt, gefragt, sein sag eigentlich beschriben und ime die bis zu gerichtlicher eröffnung zu verschweigen gebotten werden.

\section{Weliecher gestalt das $B$ gerichtsbuch, sigill und andre gerichtlich actiones bewahret werden sollen.}

Es soll des gerichts buch, sigil und was sonst an brieffen oder andrem dem gericht und der gemeinde zu Ellerstatt zustendig, in ein druglin bei einander gelegt, mit zweien schlosßen verwahrt, darzu zwen schlussel gemacht werden, deren einer der schultheiss und der ander der aeltist gerichtsscheffe haben soll. Und soll ir kheiner solich truglin one den andern eröffenen, auch nichs mit dem sigill one beisein der siben scheffen oder zum wenigsten vier auss denselben, es seie in welicherlei sachen es wölle, versiglet werden. Und deshalben soll ein iegklicher, dem etwas mit solichem gerichtssigill besigelt wurt, ein albus auch neben solichen mit schultheiss, scheffen und schreibers belohnung wie vor gemelt ist, gehalten werden. So soll auch solicher v. albus oder sigellgelt in einem seckell aufgehaben, und so der suma etwas wurt, alsdann soll schultheiss, scheffen und gerichtsschreiber soliches mit vergunstigung der obrigkheit in zeiten, so man ondas frölich sein soll, mit einander frölich und fridlich verzehren.

Und nachdem zu mehrer bestettigung und becreftigung dieser vurgenomenen gerichtsordenung ein gerichtssigil von nötten, so haben wier von obrigkheit wegen den gedachten schultheissen, scheffen und gantzer gemeind zu Ellerstatt ein gerichtssigill gehen, unserer geschlecht woppen, wie das hie beigemahlet, namblich Affensteins wappen ein bloh veld, darin ein weisser wasserstromb, und Flerssheimer woppen ist oben plo, in der miten weiss, unden rhoit. Die umbschrift des sigils soll also lauten: 
Das sigil durch die obrigkheit geben zu Ellerstatt in annum 15. 55. ${ }^{1}$ ) Das mir auch hiermit confirmieren und bestettigen thun in craft dieser ordenung also und dergestalt, das alle brieff und process, urkhunden oder verschreibungen, wie die ieder zeit genant werden mögen, mit ietztbenantem sigill zu Ellerstalt zu gezeugnus der warheit und becreftigung derselben versiglet und sollen vur angenem, gerecht und wahre confirmiert und von der obrigkheit zugelossen, bestettigt, als glaubwurdig angenomen und bei menigklichen der eren und warheit gemeß also geacht und gehalten werden, hierin alle geverd vermitten. Und dieses alles zu wahrer urkhunt haben wier Wolff von Affenstein ritter und Friederich von Flerssheim obgenant als gerichtsherren und von obrigkheit wegen dis vurgenant weissthumb, gerichtsordenung und mitgeteilten gerichtssigil $z u$ end dieser geschrifft mit eigenen handen underschriben und zu mehrer der warheit gezeugnuss und gedechtnus unser jeder sein angeborn ingesigell an ein schnur, so bloh, weiss, roit und gelh von seiden gemacht ist, durch dis aufgericht weissthumb und gerichtsordenung in der mitten durchzogen, gehangen haben.

Auf soliches alles die vurgenanten schultheiss, scheffen und gantze gemeind zu Ellerstatt mit sampt Bernhart Hamelbachen, des closters Sehbach schaffener, und Nicklos Coben, schaffener zu Schönfeldt im closter, und dann Michel Cless der muller zu Pfiffingen, so alle eigner person auch zugegen wahren und darzu sonderlichen beruffen, dieweil solches, namblich das weissthumb vornen gemelt ire verwaltente clöster

W. und muhlen berurt, noch gnungsam gehaptem bedacht sich mit antwort vernemen lossen, namblich: erstlichs der schultheiss zeigt an, wie er inhalt des wejssthumb an beden gelt, sovil das weissthumb ausswisse, nit bekhomen khunte, wolt aber derenhalben hierin khein andrung thun, sonder wölte die beden bucher und register iustificieren lossen und so er dann mangel funde, wolte er bei der herschaft umb anderung alsdann ansuchen. Noch solchem liessen die gerichtsscheffen vernemen, wie ir ietzunt nit mehr dann funfe wehren aber siben sein sollten, stunde doch bei der herschaft si zu ersetzen oder zu entsetzen, wolten dernhalben die vurgenomen werck, weliches sie vur gut, nutzlich und recht sein achten, mith nichten hinderen. Desgleichen die beide schaeffener ietztgenant der clöster Sehbach und Schoenveld zeigten an, dieweil si nichs spurten neuwes oder besonder beschwerlichs inen oder den clöstern hierin vurgenomen anders dann wie von altem hero auf si khomen, so wehren si dieser erneuwerung irer ihunckfrauwen und clöster wegen gantz wuol zufriden, wisten derowegen kheine hinderliche einred daruf zu thun. Solichem noch der muller von Pfiffingen vurbracht, er wehr ein neuw zukhommen mann, hette dieser ding aller gar nieh khein wissens gehapt, so er aber hört und befunde, das das dorf und die gemeinde zu Ellerstatt sovil gerechtigkheit auf seiner muhlen, herwiederumb auch die muhlen sovill rechts zu dem dorf und

1) Das gemalte Siegel am Rande, der Stemipel ist verloren. 
inwohnern zu Ellerstatt hette, wollte er sich mit gottes hulf aller gepur erzeigen halten und sovil im mugklich in alle weg onstraefflich finden lossen. Nach solichem allem die gantze gemeinde und ir ieder besonder mit eigenem mundt in solches alles onwiedersprechlich und einhelligklichen consentiert und gantz gutwilliglichen beiochts und angenomen, mit weiterem anzeig, si erkhennen sich irer obrigkheit in allem gehorsam zu leisten schuldig sein, haben auch die ongezweifelte dröstliche zuversicht und gute hoffnung ire herschaft werde inen oder iren nochkhommen durch dis oder dergleichen (so si jeder zeit zu thun und zu lossen haben) nichs ondaeglichs, vil weniger etwas onbillichs zumessen oder aufladen, dermhalben si solches alles gern, stedt, vest und onverbruchlich halten und hanthaben wolten, wie si mir notarii hienoch benant bei ermanung irer glupt und aeidten ein jeder besonder mit eigenen mundt versprochen und zugesagt, alles getreuwlich und ongevorlich. Gescheen am obgemelten dag, ior, monat, herschung, mohlstatt und indictio in bei und mitsein dern erbaren mit namen Jörig Dorschell, Andressen Affenstein, beide Affensteinische, und dann Hanns Muhlbrie, X. Hamns Beck und Jorig Hutt, alle drei Flersheimerische dhiener, also zu gezeugen durch ire herschaft sonderlichen hierzu geordenet.

Und dieweil ich Jaocob Wechtlin von Breißach, auß baepstlicher und Römischer kaeißerlicher macht, gewalt und begnadigung offenbarer notarius und stattschreiber zu Lampßheim noch empfangenem bevelch vornen gemelt solches alles selbs gelessen, gehört und gesehen, habe ich auf fernern empfangenen muntlichen gehaeiss solich weisthumb und gerichtsordenung hierein mit meiner eigen hant geschriben und instrumentiert und mit meinem rechten tauf und zunamen, auch gewonlichen notariatzeichen underschrieben und bezeichnet, zu glauben und wahrer urkhundt aller und ieder obgeschriebenen dingen darzuh sonderlich gebetten und beruffen.

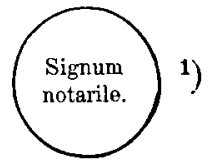

Wolff von Affenstein.

Friderich von Flersheim der iünger etc. m. pr.

Heidelberg.

Dr. Adolf Koch.

1) Das bunt gemalte Notariatszeichen trägt ausser dem Namen noch den Spruch: Salus mea Deus. 